\title{
MULTISCALE EXPANSION OF THE LATTICE POTENTIAL KDV EQUATION ON FUNCTIONS OF INFINITE SLOW-VARYNESS ORDER
}

\author{
R. HERNANDEZ HEREDERO, D. LEVI, M. PETRERA, AND C. SCIMITERNA
}

\begin{abstract}
We present a discrete multiscale expansion of the lattice potential Korteweg-de Vries (lpKdV) equation on functions of infinite order of slow-varyness. To do so we introduce a formal expansion of the shift operator on many lattices holding at all orders. The lowest secularity condition from the expansion of the lpKdV equation gives a nonlinear lattice equation, depending on shifts of all orders, of the form of the nonlinear Schrödinger (NLS) equation.
\end{abstract}

\section{INTRODUCTION}

Reductive perturbation techniques $[14,15]$ have proved to be important tools to find approximate solutions for many physical problems by reducing a given nonlinear partial differential equation to a simpler equation, which is often integrable [4]. Recently a few attempts to carry over this approach to partial difference equations have been proposed [3,6-9].

The basic tool of the discrete reductive perturbation technique developed in $[7,9]$ is a proper multiscale expansion carried out by introducing various scales and lattices defined on them. Let us recall some of the main results. The transformation between two different lattices of indices $n$ and $n_{1}$ is given by [5]

$$
\Delta_{n}^{j} u_{n} \doteq \sum_{i=0}^{j}(-1)^{j-i}\left(\begin{array}{c}
j \\
i
\end{array}\right) u_{n+i}=j ! \sum_{i=j}^{\infty} \frac{P_{i, j}}{i !} \Delta_{n_{1}}^{i} u_{n_{1}},
$$

where $u_{n}: \mathbb{Z} \rightarrow \mathbb{R}$ is a function defined on a lattice of index $n \in \mathbb{Z}$ and $u_{n_{1}}: \mathbb{Z} \rightarrow \mathbb{R}$ is the same function on a lattice of index $n_{1} \in \mathbb{Z}$. By the symbol $\Delta$ we mean the standard forward difference of the function $u$ w.r.t. its subscript, e.g. $\Delta_{n} u_{n} \doteq u_{n+1}-u_{n}$. Here the coefficients $P_{i, j}$ are given by

$$
P_{i, j} \doteq \sum_{k=j}^{i} \omega^{k} \mathcal{S}_{i}^{k} \mathfrak{S}_{k}^{j},
$$

where $\omega$ is the ratio of the increment in the lattice of variable $n_{1}$ with respect to that of variable $n$. The coefficients $\mathcal{S}_{i}^{k}$ and $\mathfrak{S}_{k}^{j}$ are the Stirling numbers of the first and second kind respectively [2]. Eq. (1.1) implies that a finite difference in the discrete variable $n$ depends on an infinite number of differences on the variable $n_{1}$, e.g. the function $u_{n+1}$ can be written as a combination of the functions $u_{i}$ 's for $i$ varying on an infinite subset of the lattice with index $n_{1}$. Formula (1.1) can be inverted yielding

$$
\Delta_{n_{1}}^{j} u_{n_{1}}=j ! \sum_{i=j}^{\infty} \frac{Q_{i, j}}{i !} \Delta_{n}^{i} u_{n}, \quad Q_{i, j} \doteq \sum_{k=j}^{i} \omega^{-k} \mathcal{S}_{i}^{k} \mathfrak{S}_{k}^{j} .
$$

In $[7,9]$ one has introduced the notion of slow-varyness of order $\ell$ for a function $u_{n}$ iff $\Delta_{n}^{\ell+1} u_{n}=$ 0 (or equivalently $\Delta_{n_{1}}^{\ell+1} u_{n_{1}}=0$, see [9] for further details). This definition enables to reduce infinite series to a finite number of terms. Moreover one has considered a generalization of formula (1.1) in order to deal with functions $u_{n}=u_{n ;\left\{n_{i}\right\}_{i=1}^{K}}$ depending on a finite number $K$ of lattice variables $n_{i}, 1 \leq i \leq K$. The computations done in [7] proved that an integrable 
lattice equation, as the lattice potential $\mathrm{KdV}(\mathrm{lpKdV})$ equation, reduces to a completely discrete nonlinear Schrödinger (dNLS) equation of the form

$$
\mathrm{i}\left(\phi_{n, m+1}-\phi_{n, m}\right)+c_{1}\left(\phi_{n+1, m}-2 \phi_{n, m}+\phi_{n-1, m}\right)+c_{2} \phi_{n, m}\left|\phi_{n, m}\right|^{2}=0,
$$

being $c_{1}, c_{2}$ two real coefficients. Let us stress the fact that the above dNLS equation has a completely local nonlinear part. We refer to the papers $[7,9]$ for further details. It has been proved by singularity confinement [13] and algebraic entropy [16] that the constructed dNLS equation is not integrable.

In [4] Calogero and Eckhaus have shown that a necessary condition for the integrability of a nonlinear partial differential equation is that its multiscale reduction be integrable. Here, trying to find a lattice analogue of the Calogero-Eckhaus theorem, we extend the techniques developed in $[7,9]$ to any order of slow-varyness.

In Section 2 we introduce a formal multiscale expansion holding at all orders of slow-varyness. Then in Section 3 we apply this technique to the lpKdV equation, thus providing an extension to all orders of the dNLS equation obtained in $[7,9]$. Finally, Section 4 is devoted to concluding remarks.

\section{Multiscale expansion on a lattice}

2.1. Lattices and shifts defined on them. Let $u_{n}: \mathbb{Z} \rightarrow \mathbb{R}$ be a function defined on a lattice of index $n \in \mathbb{Z}$. We can always extend it to a real function $u: \mathbb{R} \rightarrow \mathbb{R}$ by defining a real continuous variable $x \doteq n \sigma_{x}$, where $\sigma_{x} \in \mathbb{R}$ is the lattice spacing.

Let us define the shift operator $T_{n}$ such that $T_{n} u_{n} \doteq u_{n+1}$. For $u(x)$ we can introduce the operator $T_{x}$, corresponding to $T_{n}$, such that $T_{x} u(x) \doteq u\left(x+\sigma_{x}\right)$. The Taylor expansion of $u\left(x+\sigma_{x}\right)$ centered in $x$ reads

$$
T_{x} u(x)=u(x)+\sigma_{x} u^{(1)}(x)+\frac{\sigma_{x}^{2}}{2} u^{(2)}(x)+\ldots+\frac{\sigma_{x}^{i}}{i !} u^{(i)}(x)+\ldots=\sum_{i=0}^{\infty} \frac{\sigma_{x}^{i}}{i !} u^{(i)}(x),
$$

where $u^{(i)}(x) \doteq d^{i} u(x) / d x^{i} \doteq d_{x}^{i} u(x)$, being $d_{x}$ the total derivative operator. Eq. (2.1) suggests the following formal expansion for the differential operator $T_{x}$ :

$$
T_{x} \doteq e^{\sigma_{x} d_{x}}=\sum_{i=0}^{\infty} \frac{\sigma_{x}^{i}}{i !} d_{x}^{i} .
$$

Introducing a formal derivative with respect to the index $n$, say $\delta_{n}$, we can define, by analogy with $T_{x}$, the operator $T_{n}$ as

$$
T_{n} \doteq e^{\delta_{n}}=\sum_{i=0}^{\infty} \frac{\delta_{n}^{i}}{i !}
$$

The formal expansion (2.2) can be inverted, yielding

$$
\delta_{n}=\ln T_{n}=\ln \left(1+\Delta_{n}\right)=\sum_{i=1}^{\infty} \frac{(-1)^{i-1}}{i} \Delta_{n}^{i},
$$

where $\Delta_{n} \doteq \Delta_{n}^{+} \doteq T_{n}-1$ is the discrete first right difference operator w.r.t. the variable $n$, see eq. (1.1). Notice that this is just one of the possible inversion formulas for the operator $\delta_{n}$. For example it can be also written in terms of left difference operators $\Delta_{n}^{-} \doteq 1-T_{n}^{-1}$ :

$$
\delta_{n}=-\ln T_{n}^{-1}=-\ln \left(1-\Delta_{n}^{-}\right)=\sum_{i=1}^{\infty} \frac{\left(\Delta_{n}^{-}\right)^{i}}{i},
$$

or in terms of symmetric difference operators $\Delta_{n}^{s} \doteq\left(T_{n}-T_{n}^{-1}\right) / 2$ :

$$
\delta_{n}=\sinh ^{-1} \Delta_{n}^{s}=\sum_{i=1}^{\infty} \frac{P_{i-1}(0)}{i}\left(\Delta_{n}^{s}\right)^{i},
$$


where $P_{i}(x)$ is the $i$-th Legendre polynomial evaluated in $x=0$. Hence the $\delta_{n}$ operators are formal series containing infinite powers of $\Delta_{n}$, but, acting on slow-varying functions of order $\ell$, they reduce to polynomials in $\Delta_{n}$ of order at most $\ell$. Consequently, any formula written in terms of powers of $\delta_{n}$ for a given slow-varyness order $\ell$, contains its version for smaller orders $j<\ell$.

The convergence of the series $\delta_{n} u_{n}$ depends on the analyticity properties of the function $u_{n}$. Hence, from now on, we will proceed formally, considering that the developments can be justified a posteriori, because the functions which finally appear (solutions to the resulting difference equations) will have the correct analyticity properties.

2.2. Dilations on the lattice. Let us introduce a second lattice, obtained from the first one by a dilation. At first it is convenient to visualize the problem as a change of variable between the continuous variable $x \in \mathbb{R}$ and a new continuous variable $x_{1} \doteq \epsilon x, 0<\epsilon \ll 1$. On the lattice one considers a change from the index $n \doteq x / \sigma_{x}$ to the new index $n_{1} \doteq x_{1} / \sigma_{x_{1}}$, where $\sigma_{x_{1}}$ is the new spacing. Assuming that $\sigma_{x_{1}} \gg \sigma_{x}$ we can set $\sigma_{x} \doteq \varepsilon \sigma_{x_{1}}, 0<\varepsilon \ll 1$, so that $n_{1}=\epsilon \varepsilon n$. As $n, n_{1} \in \mathbb{Z}, \epsilon \varepsilon$ is a rational number and one can define in all generality $\epsilon \varepsilon \doteq M_{1} / N \ll 1$ with $M_{1}, N \in \mathbb{N}$. However, if we want that the lattice of index $n_{1}$ is a sublattice of the lattice of index $n$, we have also to require that $M_{1} / N=1 / M$ with $M \in \mathbb{N}$.

The relationship between the discrete derivatives defined in the two lattices is given by eq. (1.1), for which we shall give a straightforward proof based on the well-known formulas [2]

$$
\left(e^{x}-1\right)^{j}=j ! \sum_{k=j}^{\infty} \frac{\mathfrak{S}_{k}^{j}}{k !} x^{k}, \quad[\ln (1+x)]^{k}=k ! \sum_{i=k}^{\infty} \frac{\mathcal{S}_{i}^{k}}{i !} x^{i} .
$$

Here $n_{1}=n\left(M_{1} / N\right)$ and thus $\omega=M_{1} / N$. From the expression of $\Delta_{n}^{j}, j \in \mathbb{N}$, we get by a straightforward algebra:

$$
\begin{aligned}
\Delta_{n}^{j} & =\left(T_{n}-1\right)^{j}=\left(e^{\sigma_{x} d_{x}}-1\right)^{j}=j ! \sum_{k=j}^{\infty} \mathfrak{S}_{k}^{j} \frac{\left(\sigma_{x} d_{x}\right)^{k}}{k !}= \\
& =j ! \sum_{k=j}^{\infty} \frac{\mathfrak{S}_{k}^{j}}{k !}\left(\frac{\epsilon \sigma_{x}}{\sigma_{x_{1}}}\right)^{k}\left(\sigma_{x_{1}} d_{x_{1}}\right)^{k}=j ! \sum_{k=j}^{\infty} \frac{\mathfrak{S}_{k}^{j}}{k !}\left(\frac{\epsilon \sigma_{x}}{\sigma_{x_{1}}}\right)^{k}\left[\ln \left(1+\Delta_{n_{1}}\right)\right]^{k}= \\
& =j ! \sum_{k=j}^{\infty} \frac{\mathfrak{S}_{k}^{j}}{k !}\left(\frac{\epsilon \sigma_{x}}{\sigma_{x_{1}}}\right)^{k} k ! \sum_{i=k}^{\infty} \frac{\mathcal{S}_{i}^{k}}{i !} \Delta_{n_{1}}^{i}=j ! \sum_{i=j}^{\infty} \frac{1}{i !}\left[\sum_{k=j}^{i}\left(\frac{\epsilon \sigma_{x}}{\sigma_{x_{1}}}\right)^{k} \mathcal{S}_{i}^{k} \mathfrak{S}_{k}^{j}\right] \Delta_{n_{1}}^{i} .
\end{aligned}
$$

As $\sigma_{x}=\varepsilon \sigma_{x_{1}}$ and $\epsilon \varepsilon=M_{1} / N$ eq. (1.1) is proven.

2.3. Discrete multiscale expansion. We present here the formulas necessary to construct a discrete multiscale expansion. According to the definitions given above, let us consider $u \doteq$ $u_{n ; n_{1}}=u\left(x ; x_{1}\right)$ as a function depending on a fast index $n$ and a slow index $n_{1}=n\left(M_{1} / N\right)$. At the continuous level, the total derivative $d_{x}$ acting on functions $u\left(x ; x_{1}\right)$ is the sum of partial derivatives, i.e. $d_{x}=\partial_{x}+\epsilon \partial_{x_{1}}$. What is the situation on the lattice? Let us construct a relation between the total shift operator $T_{n}$ and the partial shift operators $\mathcal{T}_{n}, \mathcal{T}_{n_{1}}\left(\mathcal{T}_{n} u_{n ; n_{1}}=\right.$ $\left.u_{n+1 ; n_{1}}, \mathcal{T}_{n_{1}} u_{n ; n_{1}}=u_{n ; n_{1}+1}\right)$. As

$$
T_{x}=e^{\sigma_{x} d_{x}}=e^{\sigma_{x} \partial_{x}} e^{\epsilon \sigma_{x} \partial_{x_{1}}}
$$

we can write

with

$$
T_{n}=e^{\delta_{n}} e^{\left(M_{1} / N\right) \delta_{n_{1}}} \doteq \mathcal{T}_{n} \mathcal{T}_{n_{1}}^{\left(M_{1} / N\right)}
$$

$$
\mathcal{T}_{n} \doteq \sum_{i=0}^{\infty} \frac{\delta_{n}^{i}}{i !}, \quad \mathcal{T}_{n_{1}}^{\left(M_{1} / N\right)} \doteq \sum_{i=0}^{\infty} \frac{\left(M_{1} / N\right)^{i}}{i !} \delta_{n_{1}}^{i},
$$

where $\delta_{n_{1}}$ is given by eq. (2.3) with $n$ substituted by $n_{1}$. 
Eq. (2.4) can be easily extended to the case of $K$ slow variables $x_{i} \doteq \epsilon^{i} x, 1 \leq i \leq K$. The action of the shift operator $T_{n}$ on a function $u \doteq u_{n ;\left\{n_{i}\right\}_{i=1}^{K}}$ depending on both fast and slow variables can be written in terms of the partial shifts $\mathcal{T}_{n}, \mathcal{T}_{n_{i}}$ :

$$
T_{n} \doteq \mathcal{T}_{n} \prod_{i=1}^{K} \mathcal{T}_{n_{i}}^{\left(\epsilon_{n_{i}}\right)},
$$

where the $\epsilon_{n_{i}}$ 's are suitable functions of $\epsilon$ and $\varepsilon$ depending parametrically on some integer coefficients $M_{i} \in \mathbb{N}, 1 \leq i \leq K$.

To develop the fields appearing in partial difference equations with two independent discrete variables one has to consider the action of the operator (2.6) on a function depending on two fast indices $n$ and $m$, and on a set of $K_{n}+K_{m}$ slow variables $\left\{n_{i}\right\}_{i=1}^{K_{n}}$ and $\left\{m_{i}\right\}_{i=1}^{K_{m}}$, i.e. on

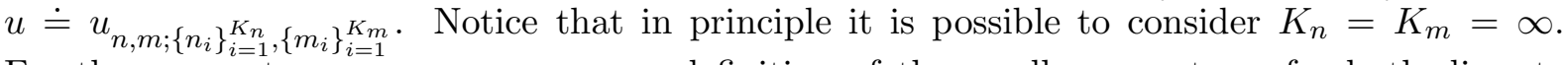
For the moment we assume a common definition of the small parameter $\epsilon$ for both discrete variables $n$ and $m$, but we denote with $M_{i}$ the integers for the slow variables $n_{i}$ and with $\widetilde{M}_{i}$ the ones for $m_{i}$. We have:

$$
\epsilon_{n_{i}} \doteq \frac{M_{i}}{N^{i}}, \quad 1 \leq i \leq K_{n}, \quad \epsilon_{m_{i}} \doteq \frac{\widetilde{M_{i}}}{N^{i}}, \quad 1 \leq i \leq K_{m}
$$

In the next Section we shall consider a partial difference equation defined on a quadrilateral lattice, namely an equation of the type $f\left(u, T_{n} u, T_{m} u, T_{n} T_{m} u\right)=0$. Assuming $K_{n}=1$ and $K_{m}=$ $K$ we get from eqs. $(2.5,2.6)$ the following expansions for the shift operators appearing in the equation $f\left(u, T_{n} u, T_{m} u, T_{n} T_{m} u\right)=0$ :

$$
\begin{aligned}
& T_{n}=\mathcal{T}_{n} \mathcal{T}_{n_{1}}^{\left(\epsilon_{n_{1}}\right)}=\mathcal{T}_{n}\left[1+\frac{1}{N} M_{1} \delta_{n_{1}}+\frac{1}{2} \frac{M_{1}^{2}}{N^{2}} \delta_{n_{1}}^{2}+\frac{1}{6} \frac{M_{1}^{3}}{N^{3}} \delta_{n_{1}}^{3}+O\left(1 / N^{4}\right)\right], \\
& T_{m}=\mathcal{T}_{m} \prod_{i=1}^{K} \mathcal{T}_{m_{i}}^{\left(\epsilon_{m_{i}}\right)}= \\
& =\mathcal{T}_{m}\left[1+\frac{1}{N} \widetilde{M}_{1} \delta_{m_{1}}+\frac{1}{N^{2}}\left(\frac{\widetilde{M}_{1}^{2}}{2} \delta_{m_{1}}^{2}+\widetilde{M}_{2} \delta_{m_{2}}\right)+\right. \\
& \left.+\frac{1}{N^{3}}\left(\frac{\widetilde{M}_{1}^{3}}{6} \delta_{m_{1}}^{3}+\widetilde{M}_{1} \widetilde{M}_{2} \delta_{m_{1}} \delta_{m_{2}}+\widetilde{M}_{3} \delta_{m_{3}}\right)+O\left(1 / N^{4}\right)\right] \text {, } \\
& T_{n} T_{m}=\mathcal{T}_{n} \mathcal{T}_{n_{1}}^{\left(\epsilon_{n_{1}}\right)} \mathcal{T}_{m} \prod_{i=1}^{K} \mathcal{T}_{m_{i}}^{\left(\epsilon_{m_{i}}\right)}= \\
& =\mathcal{T}_{n} \mathcal{T}_{m}\left[1+\frac{1}{N}\left(M_{1} \delta_{n_{1}}+\widetilde{M}_{1} \delta_{m_{1}}\right)+\right. \\
& +\frac{1}{N^{2}}\left(\frac{M_{1}^{2}}{2} \delta_{n_{1}}^{2}+M_{1} \widetilde{M}_{1} \delta_{n_{1}} \delta_{m_{1}}+\frac{\widetilde{M}_{1}^{2}}{2} \delta_{m_{1}}^{2}+\widetilde{M}_{2} \delta_{m_{2}}\right)+ \\
& +\frac{1}{N^{3}}\left(\frac{M_{1}^{3}}{6} \delta_{n_{1}}^{3}+\frac{M_{1}^{2}}{2} \widetilde{M}_{1} \delta_{n_{1}}^{2} \delta_{m_{1}}+\frac{\widetilde{M}_{1}^{2}}{2} M_{1} \delta_{n_{1}} \delta_{m_{1}}^{2}+M_{1} \widetilde{M}_{2} \delta_{n_{1}} \delta_{m_{2}}+\right. \\
& \left.\left.+\frac{\widetilde{M}_{1}^{3}}{6} \delta_{m_{1}}^{3}+\widetilde{M}_{1} \widetilde{M}_{2} \delta_{m_{1}} \delta_{m_{2}}+\widetilde{M}_{3} \delta_{m_{3}}^{3}\right)+O\left(1 / N^{4}\right)\right] .
\end{aligned}
$$




\section{Multiscale expansion of the LPKdV EQuation}

The lattice potential Korteweg-de Vries (lpKdV) equation is given by [12]:

$$
\mathcal{P} \doteq\left(p-q+u_{n, m+1}-u_{n+1, m}\right)\left(p+q-u_{n+1, m+1}+u_{n, m}\right)-\left(p^{2}-q^{2}\right)=0,
$$

where $p, q, p \neq q$, are two real parameters. The above equation is probably the best-known completely discrete nonlinear equation which involves just four points which lay on two orthogonal infinite lattices and it is nothing else but the nonlinear superposition formula for the Korteweg-de Vries equation.

By defining $\mu \doteq p-q$ and $\zeta \doteq p+q$, eq. (3.1) can be written as

$$
\mathcal{P} \doteq\left[\mu\left(T_{n} T_{m} u-u\right)+\zeta\left(T_{n} u-T_{m} u\right)\right]-\left[\left(T_{n} u-T_{m} u\right)\left(T_{n} T_{m} u-u\right)\right] \doteq \mathcal{P}_{\ell}-\mathcal{P}_{n \ell}=0,
$$

where $\mathcal{P}_{\ell}$ and $\mathcal{P}_{n \ell}$ denote respectively the linear and the nonlinear part of the lpKdV equation. The linear part $\mathcal{P}_{\ell}$ has a travelling wave solution of the form $u=\exp \{\mathrm{i}[\kappa n-\omega(\kappa) m]\}$ with

$$
\omega(\kappa)=-2 \arctan \left(\frac{\zeta+\mu}{\zeta-\mu} \tan \frac{\kappa}{2}\right) .
$$

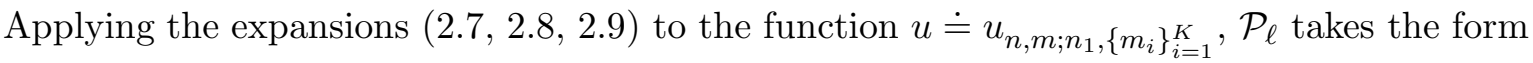

$$
\mathcal{P}_{\ell}=\sum_{i=0}^{\infty} \frac{1}{N^{i}} L_{i} u
$$

where the operators $L_{i} \doteq L_{i}\left(\mathcal{T}_{n}, \mathcal{T}_{m}, \delta_{n_{1}},\left\{\delta_{m_{j}}\right\}_{j=1}^{K}\right)$ can be constructed in a recursive way. The lowest operators $L_{i}, i=0,1,2$, read

$$
\begin{aligned}
L_{0} \doteq & \mu\left(\mathcal{T}_{n} \mathcal{T}_{m}-1\right)+\zeta\left(\mathcal{T}_{n}-\mathcal{T}_{m}\right), \\
L_{1} \doteq & \mu \mathcal{T}_{n} \mathcal{T}_{m}\left(M_{1} \delta_{n_{1}}+\widetilde{M}_{1} \delta_{m_{1}}\right)+\zeta\left(M_{1} \mathcal{T}_{n} \delta_{n_{1}}-\widetilde{M}_{1} \mathcal{T}_{m} \delta_{m_{1}}\right), \\
L_{2} \doteq & \mu \mathcal{T}_{n} \mathcal{T}_{m}\left[\frac{M_{1}^{2}}{2} \delta_{n_{1}}^{2}+M_{1} \widetilde{M}_{1} \delta_{n_{1}} \delta_{m_{1}}+\frac{\widetilde{M}_{1}^{2}}{2} \delta_{m_{1}}^{2}+\widetilde{M}_{2} \delta_{m_{2}}\right]+ \\
& +\zeta\left[\frac{M_{1}^{2}}{2} \mathcal{T}_{n} \delta_{n_{1}}^{2}-\mathcal{T}_{m}\left(\frac{\widetilde{M}_{1}^{2}}{2} \delta_{m_{1}}^{2}+\widetilde{M}_{2} \delta_{m_{2}}\right)\right] .
\end{aligned}
$$

As nonlinearity generate harmonics, let us now expand the function $u$ as

$$
u \doteq \sum_{\alpha \in \mathbb{Z}} u^{(\alpha)}\left(n_{1},\left\{m_{i}\right\}_{i=1}^{K} ; N\right) e^{\mathrm{i} \alpha(\kappa n-\omega m)} .
$$

As $u$ is assumed to be real then $u^{(-\alpha)}=\bar{u}^{(\alpha)}$, where by $\bar{u}$ we denote the complex conjugate of $u$. Moreover, if the nonlinear part should enter as a perturbation in the multiscale expansion of eq. (3.2), we need $u^{(\alpha)}\left(n_{1},\left\{m_{i}\right\}_{i=1}^{K} ; \infty\right)=0, \forall \alpha \in \mathbb{Z}$. This implies that we have to expand each function $u^{(\alpha)}$ in inverse powers of $N$ :

$$
u^{(\alpha)}\left(n_{1},\left\{m_{i}\right\}_{i=1}^{K} ; N\right) \doteq \sum_{k=1}^{\infty} \frac{1}{N^{k}} u_{k}^{(\alpha)}\left(n_{1},\left\{m_{i}\right\}_{i=1}^{K}\right) .
$$

Then $\mathcal{P}_{\ell}$ reads

$$
\mathcal{P}_{\ell}=\sum_{\alpha \in \mathbb{Z}} \sum_{i=1}^{\infty} \frac{1}{N^{i}} \sum_{k=0}^{i-1} L_{k} u_{i-k}^{(\alpha)}\left(n_{1},\left\{m_{j}\right\}_{j=1}^{K}\right) e^{\mathrm{i} \alpha(\kappa n-\omega m)} .
$$

Performing the multiscale expansion of eq. (3.2) we get several determining equations obtained selecting the different powers of $1 / N$ and the different harmonics $\alpha$.

So, let us write down the resulting determining equations at the lower orders of $1 / N$ for the harmonics $\alpha=0,1,2$, necessary to get a dNLS equation as a secularity condition. 
The order $1 / N$ gives, for $\alpha=0,1$, linear equations which are identically satisfied by taking into account the dispersion relation (3.3). For $|\alpha| \geq 2$ one gets some linear equations whose only solution is given by $u_{1}^{(\alpha)}=0$.

The order $1 / N^{2}$ gives, for the harmonics $\alpha=0,1,2$, the following equations

$$
\begin{gathered}
{\left[(\mu+\zeta) M_{1} \delta_{n_{1}}+(\mu-\zeta) \widetilde{M}_{1} \delta_{m_{1}}\right] u_{1}^{(0)}=2\left(-e^{\mathrm{i} \kappa}-e^{-\mathrm{i} \kappa}+e^{\mathrm{i} \omega}+e^{-\mathrm{i} \omega}\right)\left|u_{1}^{(1)}\right|^{2},} \\
e^{\mathrm{i} \kappa}\left(\mu e^{-\mathrm{i} \omega}+\zeta\right) M_{1} \delta_{n_{1}} u_{1}^{(1)}+e^{-\mathrm{i} \omega}\left(\mu e^{\mathrm{i} \kappa}-\zeta\right) \widetilde{M}_{1} \delta_{m_{1}} u_{1}^{(1)}=0, \\
{\left[\zeta\left(e^{2 \mathrm{i} \kappa}-e^{-2 \mathrm{i} \omega}\right)+\mu\left(e^{2 \mathrm{i}(\kappa-\omega)}-1\right)\right] u_{2}^{(2)}=\left(-e^{\mathrm{i} \kappa}+e^{-\mathrm{i} \omega}+e^{\mathrm{i}(2 \kappa-\omega)}-e^{\mathrm{i}(\kappa-2 \omega)}\right)\left(u_{1}^{(1)}\right)^{2} .}
\end{gathered}
$$

The solution of eq. (3.5) is given by $u_{1}^{(1)}\left(n_{1},\left\{m_{i}\right\}_{i=1}^{K}\right)=u_{1}^{(1)}\left(n_{2},\left\{m_{i}\right\}_{i=2}^{K}\right)$ with $n_{2} \doteq n_{1}+\gamma m_{1}$, $\gamma \doteq \mp 1$, provided that the integers $M_{1}$ and $\widetilde{M}_{1}$ are chosen as

$$
M_{1}=\gamma S e^{-\mathrm{i} \omega}\left(\mu e^{\mathrm{i} \kappa}-\zeta\right), \quad \widetilde{M}_{1}=-S e^{\mathrm{i} \kappa}\left(\mu e^{-\mathrm{i} \omega}+\zeta\right),
$$

where $S \in \mathbb{C}$ is a constant. As it has been shown in [9] one can always choose $S=r \exp (\mathrm{i} \theta)$, with $r>0$ and $\theta=-\arctan [(\zeta \sin \kappa) /(\zeta \cos \kappa-\mu)]$, in such a way that $M_{1}$ and $\widetilde{M}_{1}$ are indeed positive integers. Taking into account the dispersion relation (3.3), the coefficients $M_{1}$ and $\widetilde{M}_{1}$ in eq. (3.7) can be rewritten as

$$
M_{1}=\gamma S\left(\mu-\zeta e^{\mathrm{i} \kappa}\right), \quad \widetilde{M}_{1}=S e^{\mathrm{i} \kappa} \frac{\zeta^{2}-\mu^{2}}{\mu e^{\mathrm{i} \kappa}-\zeta} .
$$

Eqs. (3.4, 3.6) allow to express $u_{1}^{(0)}$ and $u_{2}^{(2)}$ in terms of $u_{1}^{(1)}$ and $\bar{u}_{1}^{(1)}$. As $u_{1}^{(1)}$ is a function of $n_{2}$ the same must be for $u_{1}^{(0)}$ and $u_{2}^{(2)}$, i.e. $u_{1}^{(0)}\left(n_{1},\left\{m_{i}\right\}_{i=1}^{K}\right)=u_{1}^{(0)}\left(n_{2},\left\{m_{i}\right\}_{i=2}^{K}\right), u_{2}^{(2)}\left(n_{1},\left\{m_{i}\right\}_{i=1}^{K}\right)=$ $u_{2}^{(2)}\left(n_{2},\left\{m_{i}\right\}_{i=2}^{K}\right)$. Then, taking into account eq. (3.8), we find that eqs. (3.4, 3.6) reduce respectively to

$$
\begin{array}{rlrl}
\delta_{n_{2}} u_{1}^{(0)}=\alpha_{1}\left|u_{1}^{(1)}\right|^{2}, & & \alpha_{1} \doteq-\frac{2 \gamma\left(1+e^{\mathrm{i} \kappa}\right)^{2}}{S e^{\mathrm{i} \kappa}(\mu+\zeta)\left(\mu-\zeta e^{\mathrm{i} \kappa}\right)}, \\
u_{2}^{(2)}=\alpha_{2}\left(u_{1}^{(1)}\right)^{2}, & \alpha_{2} \doteq \frac{1+e^{\mathrm{i} \kappa}}{\left(1-e^{\mathrm{i} \kappa}\right)(\mu+\zeta)} .
\end{array}
$$

We can now consider the equation for the harmonic $\alpha=1$ at order $1 / N^{3}$. We have:

$$
\begin{aligned}
& \left(\sigma_{1} \delta_{n_{1}}+\sigma_{2} \delta_{m_{1}}\right) u_{2}^{(1)}+\left(\sigma_{3} \delta_{n_{1}}^{2}+\sigma_{4} \delta_{m_{1}}^{2}+\sigma_{5} \delta_{n_{1}} \delta_{m_{1}}+\sigma_{6} \delta_{m_{2}}\right) u_{1}^{(1)}= \\
& =u_{1}^{(1)}\left(\sigma_{7} \delta_{n_{1}}+\sigma_{8} \delta_{m_{1}}\right) u_{1}^{(0)}+\sigma_{9} \bar{u}_{1}^{(1)} u_{2}^{(2)},
\end{aligned}
$$

where, taking into account eq. (3.8), the coefficients $\sigma_{i}, 1 \leq i \leq 9$, read

$$
\begin{array}{ll}
\sigma_{1} \doteq \frac{\gamma S e^{\mathrm{i} \kappa}\left(\mu-\zeta e^{\mathrm{i} \kappa}\right)\left(\mu^{2}-\zeta^{2}\right)}{\mu e^{\mathrm{i} \kappa}-\zeta}, & \sigma_{2} \doteq-\gamma \sigma_{1}, \\
\sigma_{3} \doteq \frac{1}{2} \gamma S\left(\mu-\zeta e^{\mathrm{i} \kappa}\right) \sigma_{1}, & \sigma_{4} \doteq \frac{\sigma_{1}^{2}}{2\left(\mu-\zeta e^{\mathrm{i} \kappa}\right)}, \\
\sigma_{5} \doteq-\frac{\gamma \mu \sigma_{1}^{2}}{\left(\mu^{2}-\zeta^{2}\right)}, & \sigma_{6} \doteq \widetilde{M}_{2}\left(\mu-\zeta e^{\mathrm{i} \kappa}\right), \\
\sigma_{7} \doteq \frac{\sigma_{1}\left(e^{2 \mathrm{i} \kappa}-1\right)}{e^{\mathrm{i} \kappa}(\mu+\zeta)}, & \sigma_{8} \doteq \frac{S e^{\mathrm{i} \kappa}\left(\mu^{2}-\zeta^{2}\right)(\mu+\zeta)\left(1-e^{2 \mathrm{i} \kappa}\right)}{\left(\mu e^{\mathrm{i} \kappa}-\zeta\right)^{2}}, \\
& \sigma_{9} \doteq \frac{\zeta \mu\left(e^{2 \mathrm{i} \kappa}-1\right)^{2}\left(e^{\mathrm{i} \kappa}+1\right)^{2}(\mu-\zeta)}{e^{\mathrm{i} \kappa}\left(\mu-\zeta e^{\mathrm{i} \kappa}\right)\left(\mu e^{\mathrm{i} \kappa}-\zeta\right)^{2}} .
\end{array}
$$

Using eqs. (3.9, 3.10) we can write eq. (3.11) as

$$
\left(\sigma_{1} \delta_{n_{1}}+\sigma_{2} \delta_{m_{1}}\right) u_{2}^{(1)}=\mathcal{L}\left(u_{1}^{(1)}\right)
$$


where $\mathcal{L}$ is a linear operator. Notice that the l.h.s. of eq. (3.12) is the same as in formula (3.5), but it involves the field $u_{2}^{(1)}$, instead of $u_{1}^{(1)}$. Requiring that no secular term appears we get the following equation for $u_{1}^{(1)}=u_{1}^{(1)}\left(n_{2},\left\{m_{i}\right\}_{i=2}^{K}\right)$ :

$$
\left(\sigma_{3} \delta_{n_{1}}^{2}+\sigma_{4} \delta_{m_{1}}^{2}+\sigma_{5} \delta_{n_{1}} \delta_{m_{1}}+\sigma_{6} \delta_{m_{2}}\right) u_{1}^{(1)}=u_{1}^{(1)}\left(\sigma_{7} \delta_{n_{1}}+\sigma_{8} \delta_{m_{1}}\right) u_{1}^{(0)}+\sigma_{9} \bar{u}_{1}^{(1)} u_{2}^{(2)} .
$$

Then from eq. (3.12) we see that $u_{2}^{(1)}$ will satisfy the equation $\left(\sigma_{1} \delta_{n_{1}}+\sigma_{2} \delta_{m_{1}}\right) u_{2}^{(1)}=0$, i.e. $u_{2}^{(1)}\left(n_{1},\left\{m_{i}\right\}_{i=1}^{K}\right)=u_{2}^{(1)}\left(n_{2},\left\{m_{i}\right\}_{i=2}^{K}\right)$ whenever eq. (3.8) holds. Using eqs. (3.9, 3.10) we find that eq. (3.13) is equivalent to the dNLS equation

$$
\mathrm{i} \delta_{m_{2}} u_{1}^{(1)}=\rho_{1} \delta_{n_{2}}^{2} u_{1}^{(1)}+\rho_{2} u_{1}^{(1)}\left|u_{1}^{(1)}\right|^{2},
$$

where

$$
\begin{aligned}
& \rho_{1} \doteq \frac{\mathrm{i} \zeta \mu S^{2} e^{\mathrm{i} \kappa}\left(\zeta^{2}-\mu^{2}\right)\left(e^{2 \mathrm{i} \kappa}-1\right)}{2 \widetilde{M}_{2}\left(\mu e^{\mathrm{i} \kappa}-\zeta\right)} \\
& \rho_{2} \doteq \frac{\mathrm{i} \zeta \mu(\mu-\zeta)\left(e^{2 \mathrm{i} \kappa}-1\right)\left(e^{\mathrm{i} \kappa}+1\right)^{4}}{\widetilde{M}_{2} e^{\mathrm{i} \kappa}(\mu+\zeta)\left(\mu e^{\mathrm{i} \kappa}-\zeta\right)^{2}\left(\zeta e^{\mathrm{i} \kappa}-\mu\right)^{2}}
\end{aligned}
$$

Taking into account the form of $S$, one finds that $\rho_{1}$ and $\rho_{2}$ are both real numbers:

$$
\begin{aligned}
\rho_{1} & =\frac{\mu \zeta r^{2}\left(\zeta^{2}-\mu^{2}\right) \sin \kappa}{\widetilde{M}_{2}\left(\zeta^{2}+\mu^{2}-2 \zeta \mu \cos \kappa\right)}, \\
\rho_{2} & =-\frac{8 \zeta \mu(\zeta-\mu)(1+\cos \kappa)^{2} \sin \kappa}{\widetilde{M}_{2}(\mu+\zeta)\left(\zeta^{2}+\mu^{2}-2 \zeta \mu \cos \kappa\right)^{2}} .
\end{aligned}
$$

Notice that $\rho_{1} \rho_{2}<0$, so that eq. (3.14) is a defocusing dNLS equation.

Eq. (3.14) may be obtained as a compatibility condition of the linear problem

$$
\begin{aligned}
& \delta_{n_{2}} \Phi_{n_{2}, m_{2}}(\eta)=U\left(u_{1}^{(1)}, \bar{u}_{1}^{(1)} ; \eta\right) \Phi_{n_{2}, m_{2}}(\eta), \\
& \delta_{m_{2}} \Phi_{n_{2}, m_{2}}(\eta)=V\left(u_{1}^{(1)}, \bar{u}_{1}^{(1)} ; \eta\right) \Phi_{n_{2}, m_{2}}(\eta),
\end{aligned}
$$

with

$$
\begin{aligned}
U\left(u_{1}^{(1)}, \bar{u}_{1}^{(1)} ; \eta\right) & \doteq\left(\begin{array}{cc}
\mathrm{i} \eta & u_{1}^{(1)} \\
\bar{u}_{1}^{(1)} & -\mathrm{i} \eta
\end{array}\right), \\
V\left(u_{1}^{(1)}, \bar{u}_{1}^{(1)} ; \eta\right) & \doteq\left(\begin{array}{cc}
2 \mathrm{i} \eta^{2}+\mathrm{i}\left|u_{1}^{(1)}\right|^{2} & 2 \eta u_{1}^{(1)}-\mathrm{i} \delta_{n_{2}} u_{1}^{(1)} \\
2 \eta \bar{u}_{1}^{(1)}+\mathrm{i} \delta_{n_{2}} \bar{u}_{1}^{(1)} & -2 \mathrm{i} \eta^{2}-\mathrm{i}\left|u_{1}^{(1)}\right|^{2}
\end{array}\right),
\end{aligned}
$$

being $\eta \in \mathbb{C}$ the spectral parameter and $\Phi_{n_{2}, m_{2}}(\eta)$ a discrete complex vector function. A necessary and sufficient condition for the integrability of eq. (3.14) is that the coefficients $\rho_{1}$ and $\rho_{2}$ be real. Thus eq. (3.14) is integrable iff $\ell=\infty$.

\section{Concluding Remarks}

In this paper we have extended the results obtained in $[7,9]$ to the case of functions of slowvaryness of order infinity. To do so we have used the connection between shift operators and infinite series of differential operators (2.2). Using these formulas we have been able to easily recover the formula (1.1), usually proved by combinatorial techniques [5]. Moreover, in analogy with the continuous case we can introduce partial shift operators, which are expressed in terms of $\delta$ operators, namely infinite series of difference operators.

The multiscale expansion of the lpKdV equation in terms of harmonics implies, as a condition for the non existence of secular terms, a NLS equation written in terms of $\delta$ operators. Choosing $\delta_{n_{2}}$ in eq. (3.14) as a series of symmetric differences and fixing $\ell=2$ the resulting dNLS equation is equivalent to the one presented in $[7,9]$, see eq. (1.2). In this way we have 
shown that for any finite order of slow-varyness the obtained dNLS equation will be local and no summation terms will ever appear.

As shown in Section 3, eq. (3.14) has a matrix Lax pair (3.15, 3.16), which is also expressed in terms of $\delta$ operators. Such Lax pair has a reduction to any finite order of slow-varyness, provided that the associated wave functions $\Phi_{n_{2}, m_{2}}(\eta)$ have the same finite order of slow-varyness. However in the case of finite $\ell$ the Lax pair (3.15, 3.16) reduces to difference equations and their compatibility is no more satisfied by the slow-varying approximation of eq. (3.14). In fact the difference operators $\Delta$ do not satisfy the Leibniz rule.

This work still leaves many open problems on which we are working at the moment. Let us just mention the analysis of the solutions of the lpKdV equation obtained from the exact ones of the reduced equation and their comparison with the ones obtained by carrying out a multiscale expansion of the continuous potential $\mathrm{KdV}$ equation; the reduction of symmetries of the lpKdV equation; the reduction of differential-difference equations both integrable and nonintegrable.

It is still an open problem to understand the role played by an integrable discrete equation like the nonlocal discrete NLS equation introduced by Ablowitz and Ladik [1] from the isospectral compatibility of a discrete analogue of the Zakharov-Shabat spectral problem (3.15, 3.16):

$$
\begin{aligned}
\mathrm{i} \frac{\Delta_{m} u_{n, m}}{\Delta t} & =\frac{1}{2(\Delta x)^{2}}\left[\left(u_{n+1, m}-2 u_{n, m}+u_{n-1, m} \prod_{k=-\infty}^{n-1} \Lambda_{k, m}\right)+\right. \\
& \left.+\left(u_{n+1, m+1} \prod_{k=-\infty}^{n} \Lambda_{k, m}-2 u_{n, m+1}+u_{n-1, m+1}\right)\right] \pm \\
& \pm \frac{1}{4}\left[u_{n, m}\left(\bar{u}_{n, m} u_{n+1, m}+\bar{u}_{n, m+1} u_{n+1, m+1}\right)+\right. \\
& +u_{n, m+1}\left(u_{n-1, m} \bar{u}_{n, m}+u_{n-1, m+1} \bar{u}_{n, m+1}\right)+ \\
& \left.+2\left|u_{n, m}\right|^{2} u_{n+1, m+1} \prod_{k=-\infty}^{n} \Lambda_{k, m}+2\left|u_{n, m+1}\right|^{2} u_{n-1, m} \prod_{k=-\infty}^{n-1} \Lambda_{k, m}\right]- \\
& -u_{n, m} \sum_{k=-\infty}^{n} \Delta_{m} S_{k, m}-u_{n, m+1} \sum_{k=-\infty}^{n-1} \Delta_{m} \bar{S}_{k, m},
\end{aligned}
$$

with

$$
\Lambda_{k, m} \doteq \frac{1 \pm\left|u_{k, m+1}\right|^{2}}{1 \pm\left|u_{k, m}\right|^{2}}, \quad S_{k, m} \doteq u_{k, m} \bar{u}_{k-1, m}+u_{k+1, m} \bar{u}_{k, m}
$$

Eq. (4.1) reduces to the continuous NLS equation when $\Delta t \rightarrow 0$ and $\Delta x \rightarrow 0$

\section{ACKnowledgments}

RHH was partially supported by the Region of Madrid and Universidad Politécnica de Madrid (UPM) through the grant ref. CCG06-UPM/MTM-539. DL and MP were partially supported by PRIN Project SINTESI-2004 of the Italian Minister for Education and Scientific Research. MP was partially supported by the European Community through the FP6 Marie Curie RTN ENIGMA (contract number MRTN-CT-2004-5652).

\section{REFERENCES}

[1] Ablowitz M.J. and Ladik J.F., Stud. Appl. Math. 55 (1976) 1011-1018.

[2] Abramowitz M. and Stegun I.A., Handbook of mathematical functions with formulas, graphs, and mathematical tables, Dover Publications, Inc., New York, 1992.

[3] Agrotis M., Lafortune S. and Kevrekidis P.G., Discr. Cont. Dyn. Sist. 2005 supp. 22-29.

[4] Calogero F. and Eckhaus W., Inv. Prob. 32 (1987) 229-262.

Calogero F. and Eckhaus W., Inv. Prob. 41 (1987) 11-33.

[5] Jordan C., Calculus of finite differences, Röttig and Romwalter, Sopron, 1939. 
[6] Leon J. and Manna M., Journ. Phys. A 32 (1999) 2845-2869.

[7] Levi D., Jour. Phys. A 38 (2005) 7677-7685.

[8] Levi D. and Heredero R., Jour. Nonlinear Math. Phys. 121 (2005) 440-455.

[9] Levi D. and Petrera M., Jour. Math. Phys. 47 (2006) 043509.

[10] Levi D. and Petrera M., Jour. Phys. A 40 (2007) 4141-4159.

[11] Levi D. and Winternitz P., Jour. Phys. A 39 (2006) R1-R63.

[12] Nijhoff F.W. and Capel H.W., Acta Appl. Math. 39 (1995) 133-158.

[13] Papageorgiou V.G., private communication.

[14] Taniuti T., Prog. Theor. Phys. 551 (1974) 1654-1676.

[15] Taniuti T. and Nishihara K., Nonlinear waves, Pitman, Boston, 1983.

[16] Viallet C., private communication.

Universidad Politécnica de Madrid, Escuela Universitaria de Ingeniería Técnica de Telecomunicación, Departamento de Matemática Aplicada, Campus Sur Ctra de Valencia Km. 728031, MADRID, SPAIN

E-mail address: rafahh@euitt.upm.es

Dipartimento di Ingegneria Elettronica, Università degli Studi Roma Tre and Sezione INFN, Roma Tre, Via della Vasca Navale 84, 00146 Roma, Italy

E-mail address: levi@fis.uniroma3.it

Zentrum Mathematik, Technische Universität München, Boltzmannstr. 3, D-85747 Garching Bei München, Germany, and Dipartimento di Ingegneria Elettronica, Università degli Studi Roma Tre, Via della Vasca Navale 84, 00146 Roma, Italy

E-mail address: petrera@ma.tum.de

Dipartimento di Fisica e Dipartimento di Ingegneria Elettronica, Università degli Studi Roma Tre and Sezione INFN, Roma Tre, Via della Vasca Navale 84, 00146 Roma, Italy

E-mail address: scimiterna@fis.uniroma3.it 